\title{
Le silex du Flysch de Montgaillard et son exploitation sur les ateliers du Paléolithique supérieur à Hibarette (Hautes-Pyrénées)
}

The flint of the Montgaillard flysch and its production on the Upper Palaeolithic workshops in Hibarette (Hautes-Pyrénées)

Jean Barragué, Élisabeth Barragué, Marc Jarry, Pascal Foucher et Robert Simonnet

\section{OpenEdition}

Journals

Édition électronique

URL : http://journals.openedition.org/paleo/951

DOI : 10.4000/paleo.951

ISSN : 2101-0420

Éditeur

SAMRA

Édition imprimée

Date de publication : 14 décembre 2001

Pagination : 29-52

ISSN : 1145-3370

Référence électronique

Jean Barragué, Élisabeth Barragué, Marc Jarry, Pascal Foucher et Robert Simonnet, « Le silex du

Flysch de Montgaillard et son exploitation sur les ateliers du Paléolithique supérieur à Hibarette

(Hautes-Pyrénées) », PALEO [En ligne], 13 | 2001, mis en ligne le 26 mai 2010, consulté le 10 décembre 2020. URL : http://journals.openedition.org/paleo/951 ; DOI : https://doi.org/10.4000/paleo.951

Ce document a été généré automatiquement le 10 décembre 2020.

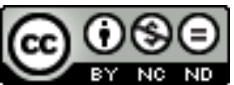

PALEO est mis à disposition selon les termes de la licence Creative Commons Attribution - Pas d'Utilisation Commerciale - Pas de Modification 4.0 International. 


\section{Le silex du Flysch de Montgaillard et son exploitation sur les ateliers du Paléolithique supérieur à Hibarette (Hautes-Pyrénées)}

The flint of the Montgaillard flysch and its production on the Upper Palaeolithic workshops in Hibarette (Hautes-Pyrénées)

Jean Barragué, Élisabeth Barragué, Marc Jarry, Pascal Foucher et Robert Simonnet

À la mémoire de Thierry Barragué

\section{Introduction}

1 En recherchant l'origine des silex utilisés dans une grotte des environs de Bagnères-deBigorre, E. et Ch. Frossard, en 1869, remarquent que la plupart provient d'affleurements crétacés qui passent à $9 \mathrm{~km}$ en aval du site. Les auteurs ont vu à Noudrest (Montgaillard) «des silex en bancs et rognons peu considérables et pas toujours d'assez bonne qualité pour être taillés » (Frossard, Frossard $1880: 20$ ). Il s'agit alors de la carrière Dussert à Montgaillard.

2 La présence de silex est donc connue de longue date dans cette région du piémont pyrénéen. L'intérêt pour ce matériau a été relancé par les découvertes faites par Jean et Thierry Barragué ${ }^{\dagger}$ dans les années 1980 (Clottes 1985 et 1989). En effet, plusieurs concentrations de blocs de silex bruts accompagnés d'un important matériel archéologique sont identifiées sur les hauteurs dominant le village d'Hibarette. Le site livre alors aux prospecteurs d'énormes quantités de vestiges. Par ailleurs, ce type de silex était déjà identifié dans les séries lithiques d'habitats magdaléniens dans les 
grottes de la région (notamment $c f$. Simonnet 1991). Ce nouveau gîte et son exploitation ont été accessoirement mentionnés à plusieurs reprises dans des notes.

Une partie de l'industrie a déjà été décrite par l'un de nous (Jarry 1992). Le présent article livre les résultats des derniers travaux effectués sur ce gisement de silex, augmentés des récoltes des prospections récentes.

\section{Le Silex De Montgaillard/Hibarette}

\section{Les gîtes}

Les gîtes paraissent relativement isolés dans le contexte nord pyrénéen. Ils sont à une trentaine de kilomètres plus à l'est des gîtes du flysch prospectés par $\mathrm{S}$. Lacombe et $\mathrm{Ch}$. Normand, et à moins de $50 \mathrm{~km}$ du massif de Montmaurin-Lespugue où s'achève l'ensemble des Prépyrénées (fig. 1).

Figure 1 -Situation géographique des gîtes/ateliers d'Hibarette dans le Grand Sud-Ouest (carte de M. Jarry).

Figure 1 - Location of the Hibarette flint workshops in Grand Sud-Ouest.

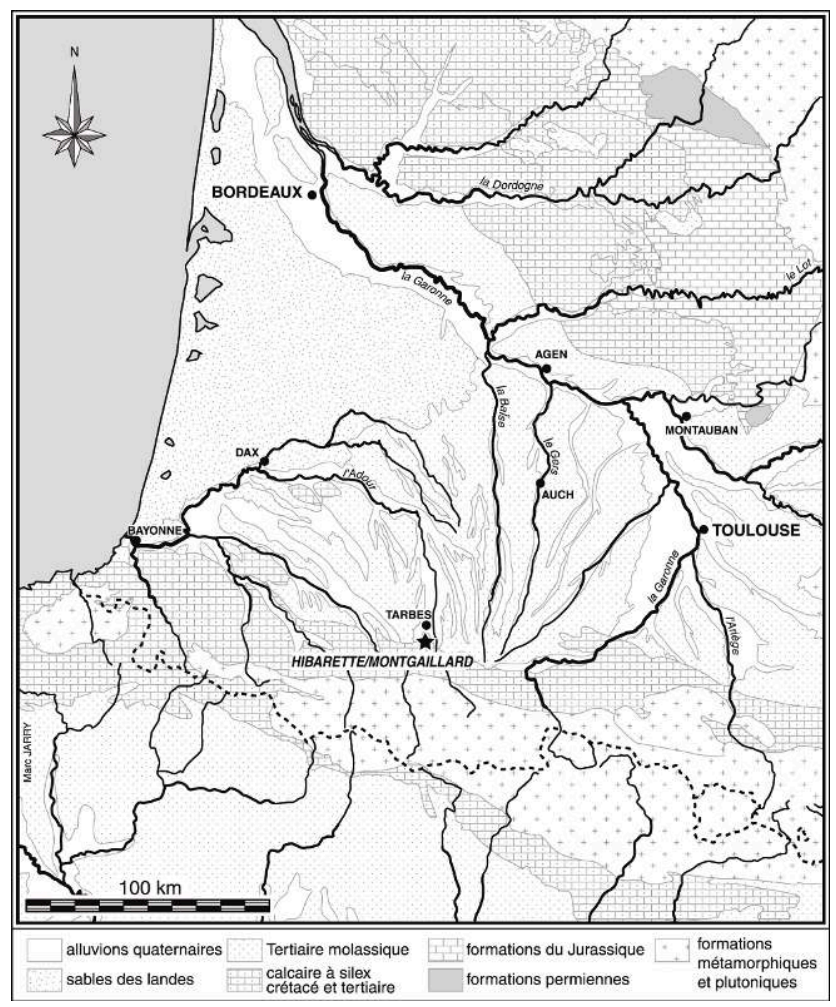

Les concentrations de blocs et d'industries se répartissent dans un espace d'environ 1,5 $\mathrm{km}$ sur $800 \mathrm{~m}$ sur les communes de Bénac, Hibarette, Saint-Martin et Visker. Le nom d'Hibarette a été retenu pour désigner l'ensemble. Les lieux-dits sont : sur la hauteur, Coustaret, Le Bois du Bécut et Las Vignes, sur la pente est, vers l'Adour, Bioues et Pého, sur la pente ouest la Décharge et enfin, en fond de vallée, sur la terrasse de l'Aube, Las Sablas (fig. 2). La Décharge est une immense tranchée creusée, depuis le plateau jusqu'à la plaine, par la ville de Tarbes pour y déposer les ordures ménagères. Une partie du site a donc été détruite, il reste cependant de longues et hautes coupes qui sont les seuls regards sur les terrains affleurants et la sédimentation. Les nodules de silex, en 
surface, sont mélangés aux blocs de calcaire et à d'autres roches. Aucune industrie n'a été remarquée en profondeur dans les sédiments fins, ce qui laisse supposer, ici comme ailleurs, que tout est en surface ou presque.

Figure 2 - Localisation des gîtes/ateliers d'Hibarette sur carte altimétrique (gauche) et carte géologique (droite) (cartes de M. Jarry)

Figure 2 - Location of the Hibarette flint workshops on altimetric map (left) and geologic map (right).

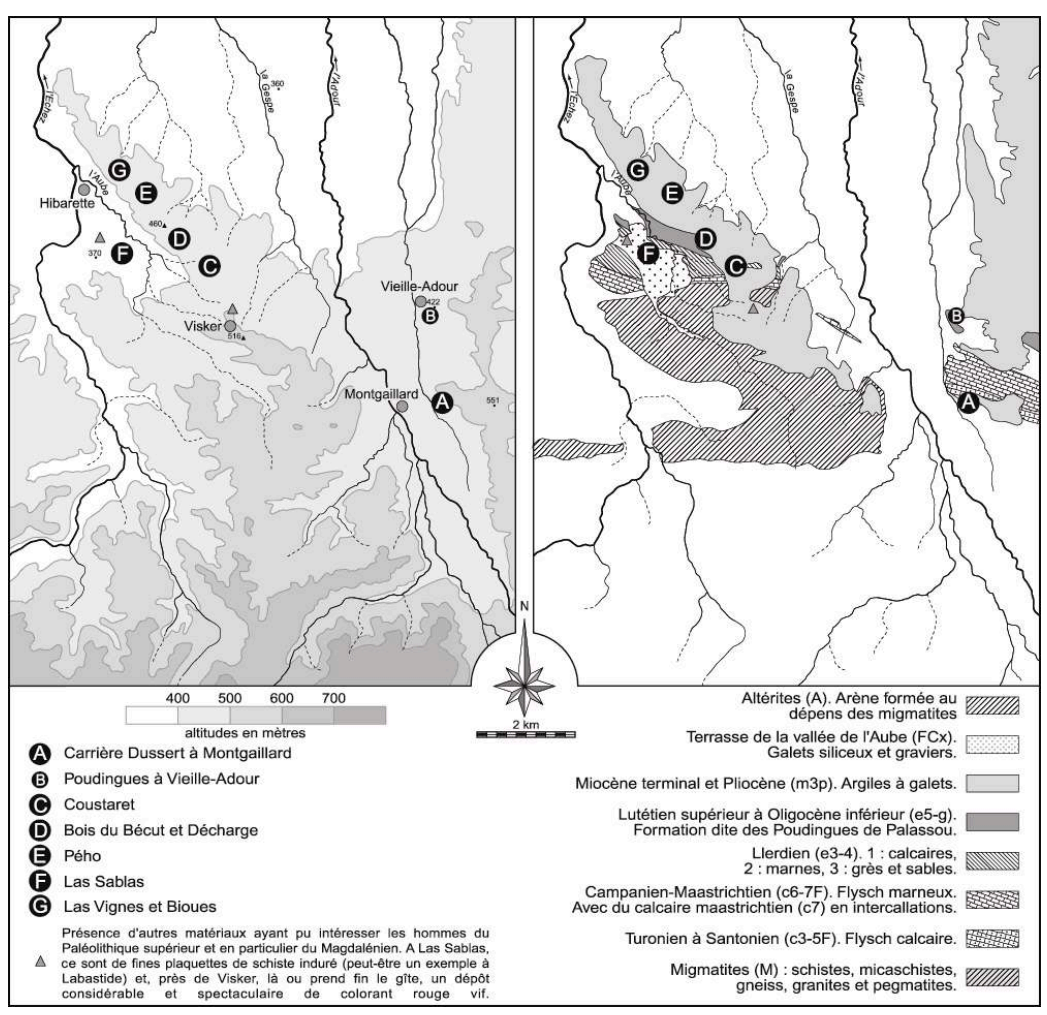

La dénivellation entre le point le plus haut et Las Sablas, au fond de la vallée de l'Aube, est d'une centaine de mètres. Coustaret est à $480 \mathrm{~m}$ c'est-à-dire à une altitude qui correspond aux cas extrêmes enregistrés dans les Prépyrénées (Le Pla, Couteret, Tucaou) où la moyenne évolue entre 350 et $400 \mathrm{~m}$. Les Prépyrénées sont nettement dégagées des altitudes proprement montagnardes et s'en écartent encore plus à l'ouest de la cluse de Boussens. Hibarette est dans une localisation bien différente ; on est au début du glacis qui, en moins de $25 \mathrm{~km}$, conduit aux $2872 \mathrm{~m}$ du Pic du Midi. Or, Coustaret a été fréquenté par les Solutréens puisque c'est de là que provient le plus grand nombre de feuilles de laurier du site. Il y a donc, en ce point des Pyrénées, un repère à prendre en compte dans la cartographie des espaces habitables au maximum glaciaire.

7 L'ensemble d'Hibarette est un gîte secondaire. A l'exception de Las Sablas ${ }^{1}$, dont le dépôt est de formation plus récente, les blocs de silex sont dans la dépendance d'une nappe détritique correspondant à "la sédimentation torrentielle du Miocène supérieur" cartographiée m3-p (fig. 2) ayant fait suite à "un épisode de creusement des vallées" consécutif au dernier soulèvement de la chaîne.

Ces blocs sont issus des silicifications en gite primaire des calcaires du flysch dont fait partie l'affleurement situé à Montgaillard, au silex déjà connu et daté du Crétacé supérieur. Deux grandes formations sont figurées sur la carte géologique et décrites dans la notice (fig. 2). Il s'agit des références "c2-3F, Cénomanien moyen à Turonien, 
flysch gris." et "c6-7F, Campanien-Maastrichtien. Flysch marneux.”. La carrière Dussert est citée dans la deuxième de ces formations "calcaires maastrichtiens (notés C7) qui sont surtout visibles dans une ancienne carrière au nord du Soum de Buala, le long de la route départementale 28 .". Or d'après la carte, cette carrière est située dans la première formation. L'absence de critères biostratigraphiques dans les échantillons de silex ne permet pas de trancher entre "Turonien à Santonien" et "Campanien-Maastrichtien" (tabl. 1: lames minces C 30184, C 30185, C 30186). La référence à "silex type Montgaillard-Hibarette" évite d'entrer dans le débat, l'essentiel étant d'identifier le matériau suffisamment caractérisé par d'autres aspects et d'en localiser l'origine.

Tableau 1 - Analyse des lames minces.

Table 1 - Analysis of the thin sections.

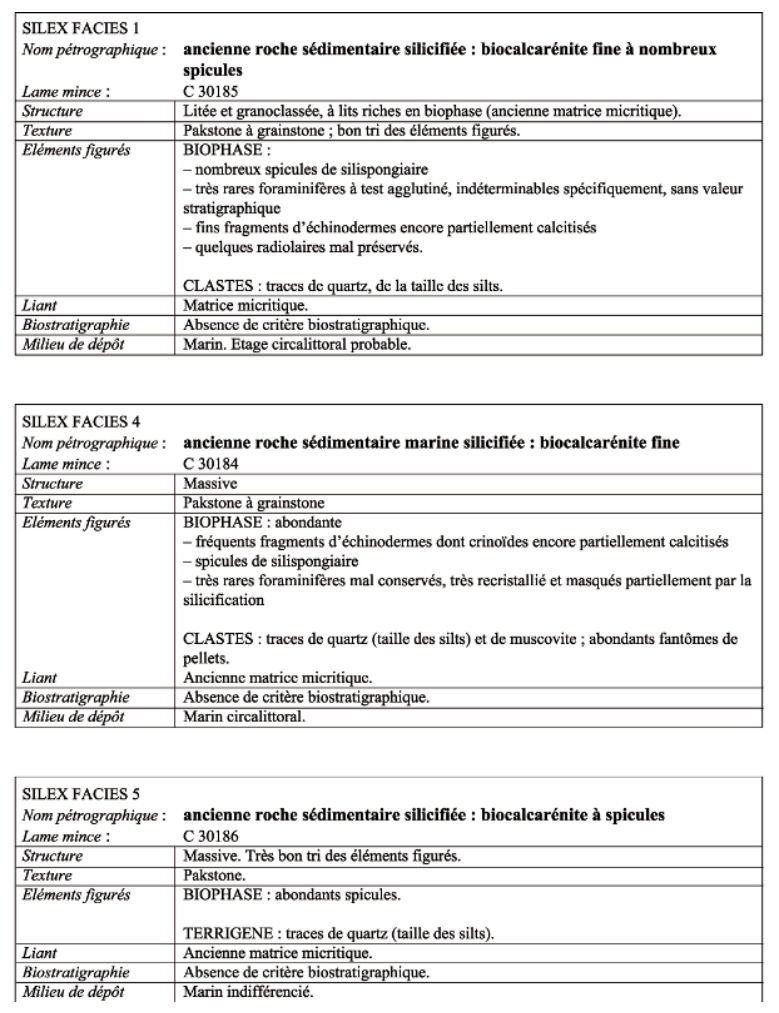

D'après ce qu'il a été possible d'observer à différentes dates sur les coupes encore visibles de la Décharge, il parait probable que les matériaux détritiques décrits par la notice géologique sous la référence e5-g proviennent du démantèlement de la formation déjà détritique que sont les poudingues de Palassou. Le gîte d'Hibarette serait, si on peut dire, deux fois secondaire, la position primaire étant dans le contexte des bancs calcaires dont la référence est en affleurement à Montgaillard. La preuve de ce transit possible, en position intermédiaire, par les poudingues de Palassou est apportée par le pointement de ces derniers dans le village même de Vielle-Adour où on remarque, cimentés parmi les blocs calcaires, des silex dont le gîte primaire est à 1,5 $\mathrm{km}$ en amont à Montgaillard. Cependant, on note qu'à la Décharge, les blocs de calcaire sont de dimensions variables, souvent gros et, conformément à la description de la notice géologique qui ignorait ce regard dans l'épaisseur des sédiments, il n'y est pas question de silex. Le seul silex qu'on y observe est encore pris, non dégagé comme en position primaire, dans la masse du calcaire de quelques blocs; aucun nodule n'a été remarqué en profondeur dans ces coupes. Comme il a été précisé plus haut, il se 
confirme donc que tout est bien en surface dans la formation détritique la plus récente. Ceci n'exclut pourtant pas un emprunt, légèrement en amont, à un niveau particulier des poudingues dont la barre affleurante est à la Décharge, ici redressée à $40^{\circ}$, et inconnu ou non décrit par les géologues. Il ne faut pas perdre de vue que les géologues ont rarement eu accès, dans ce massif, à des "regards" en profondeur.

\section{Les caractères intrinsèques de la matière première}

Les blocs de silex libérés à Hibarette se présentent sous les formes qu'ils ont en gîtes primaires telles qu'on peut les voir dans les fronts étagés du calcaire de la carrière à Montgaillard: en nodules arrondis plus ou moins gros (dont un silex noir dans une vaste poche de calcaire noir), en plaquettes d'épaisseurs variables.

11 Les cortex, réguliers et fins, sont aussi d'épaisseurs variables, de $1 \mathrm{~mm}$ à $20 \mathrm{~mm}$. Les défauts, surtout sur les plaquettes, ont créé, après fragmentations naturelles, des surfaces planes plus ou moins altérées.

La couleur largement dominante va du gris au brun. Le noir est souvent associé au brun (tabl. 1 : lame mince C 30184), en variabilité intrabloc: cœur noir sous une épaisseur variable brune (après débitage, un même bloc peut se cacher sous trois types de silex). Selon le degré d'altération, la couleur des artefacts passe d'un gris bleuté à un brun crème qui est largement dominant.

Fig. 3 - Exemples de variabilité du silex de Montgaillard/Hibarette (caractères pétrographiques et altérations).

Fig. 3 - Samples of the Montgaillard/Hibarette flint variability (petrographic characters and alterations).
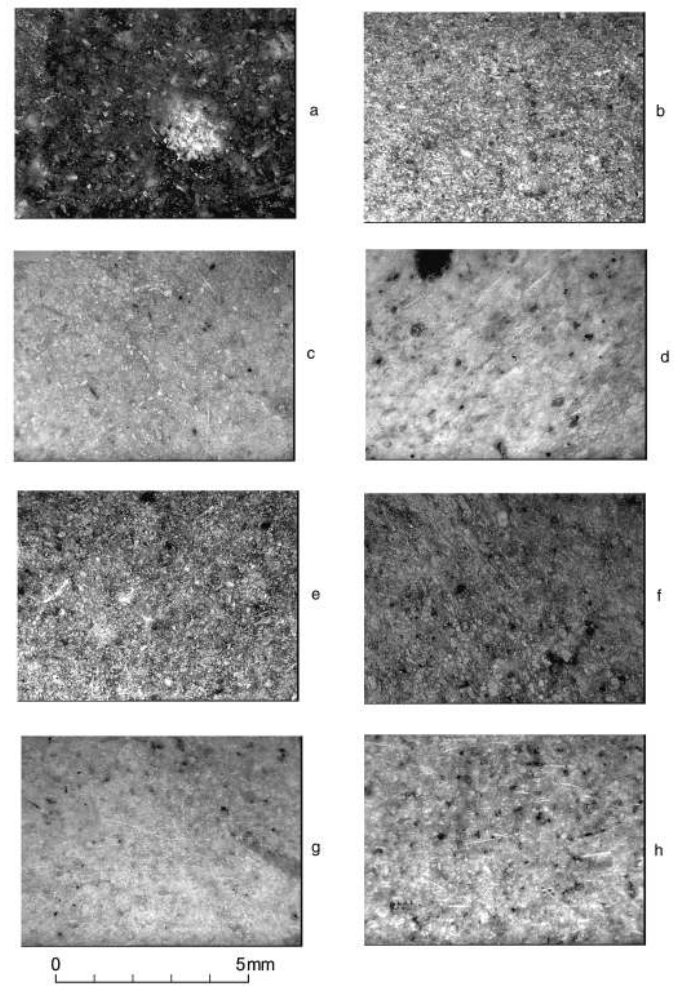

Clichés photographiques à grossissement $x 10$ (chaque graduation de la règle correspond à $1 \mathrm{~mm}$ ). Photomicrograph : magnification $\times 10$.

[Clichés P. Chalard et P. Foucher]. 
Les intraclastes associés semblent assez caractéristiques malgré le flou des attributions stratigraphiques, voire leur absence :

- abondants grains ovoïdes (pellets) parfaits ou plus ou moins défaits selon les zones, de couleur blanchâtre ; les groupements en pelotes qui se remarquent dans certaines zones paraissent un bon critère (fig. 3a) ;

- "en semis", corps défaits hauts en couleurs : jaunes, jaunes-rouille, rouille pour le brun, noirs pour le noir ;

- par zones, forte densité de spicules de silispongiaires alignés (fig. 3h). Rares petits foraminifères benthiques identifiables (fig. 3d) ;

- quartz clastiques miroitant sur les surfaces éclatées ;

- impuretés carbonatées (irisées) parfois grosses, attaquées à l'acide en test destructeurs laissant des vacuoles.

Lorsque l'altération graphique est intervenue, les intraclastes restent toujours perceptibles, par leurs contours et leurs couleurs, sur les surfaces de débitage des industries du gîte et des habitats plus ou moins lointains. Cela donne une sorte de léger moucheté de grains blancs et de couleur, avec parfois des zones où la densité des spicules devient plus élevée (fig. 3).

De son côté, l'étude de l'industrie a isolé une variété de silex que l'usage appellerait "silex blond". Ce matériau a attiré l'attention par sa ressemblance avec un des types courants de silex du Turonien au Grand-Pressigny auquel on pense au premier abord. En supposant, bien entendu, une source inconnue plus proche que celle évoquée, l'éventualité d'un type supplémentaire de silex exogène ne pouvait donc être écartée $a$ priori jusqu'à ce que la découverte récente d'un bloc volumineux apporte la preuve qu'il s'agit bien d'un type particulier, peu fréquent mais appartenant bien au gite. Par la finesse de son cortex, la couleur et la texture de son silex, ce bloc a confirmé la ressemblance trompeuse. A son niveau d'analyse et d'après sa fiche descriptive, la lame mince ne paraît pas apporter d'informations déterminantes; il reste que le Turonien est présent à Montgaillard, malheureusement mal localisé pour notre usage.

Toujours dans l'industrie, il a été remarqué un autre cas particulier susceptible d'entraîner, au-dessous d'une certaine maille de débitage, une erreur d'attribution. Il s'agit d'un silex à structure brèchoïde rappelant un des caractères du grand traceur qu'est le matériau du gîte du Verdier, lui aussi bien lointain. Il n'a pas encore été trouvé de bloc brut identique. Cependant, on est certain qu'on a affaire au moins à un cas de variabilité intra-bloc; en effet un grand éclat présente localement cette structure puis une zone de passage au terme de laquelle on retrouve les caractéristiques du silex de Montgaillard-Hibarette.

Enfin, et toujours en ce qui concerne les risques d'erreur, il importe de signaler un cas limite d'altération qui, sans une bonne connaissance des deux gîtes, pourrait prêter à confusion avec l'altération mouchetée du gîte du Paillon à Saint-Martory (HauteGaronne).

\section{L'industrie lithique des ateliers}

18 L'importante quantité de silex taillés ramassés sur une vingtaine d'années et la nature du site (de surface) ont été des facteurs déterminants dans l'orientation de notre 
étude ; traiter plus d'une tonne d'industrie lithique n'est pas sans poser de problèmes et la restitution des données en a été forcément adaptée.

Le site principal a été divisé en quatre grandes zones contiguës (zones 1 à 4 se localisant au point $\mathrm{D}$ Bois de Bécut), individualisées au cours des prospections en fonction de la dispersion et des concentrations de matériel qui sont apparues. Ainsi, chaque grand ensemble lithique issu de ces zones a fait l'objet d'une étude séparée.

La première étude qui avait été faite sur cette collection avait démontré qu'elle recoupait plusieurs grandes périodes de la Préhistoire (Jarry 1992). De par la présence de pièces caractéristiques (typologiques ou technologiques), on pouvait y déceler du Paléolithique moyen, du Paléolithique supérieur et du Néolithique. Face à ces mélanges manifestes, nous avons opéré un tri préalable dans l'industrie en écartant tout ce qui se rapportait à l'évidence au Paléolithique moyen ${ }^{2}$ et au Néolithique. Le résultat a été de constituer un ensemble d'étude, forcément artificiel, qui devait rassembler les principales composantes de l'industrie du Paléolithique supérieur; nous avons tenté par la suite de les mettre en évidence et de les caractériser par le biais d'une analyse typologique et technologique restreinte (étude des nucléus).

\section{Quelques données d'ordre typologique sur l'outillage}

\section{Zone 1}

L'effectif total des outils de la zone 1 s'élève à 1205 (tabl. 2). L'importance numérique des lames et des éclats retouchés tend à écraser les autres catégories d'outils. Cette donnée peut être expliquée par la fonction du site qui devait être avant tout un atelier de production laminaire; les lames débitées à profusion sur le site, qui n'étaient pas destinées à être emportées, semblent avoir été majoritairement employées pour les activités annexes des préhistoriques. 
Tableau 2 - Décompte des outils par zones (tous types de silex). Table 2 - Tools counts of the differents areas.

\begin{tabular}{|c|c|c|c|c|c|}
\hline Types d'outils & Zor & ne 1 & Zone 2 & Zone 3 & Zone 4 \\
\hline 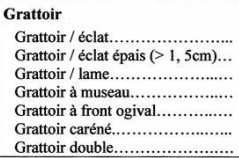 & \begin{tabular}{|l|}
173 \\
76 \\
12 \\
40 \\
5 \\
2 \\
38 \\
\end{tabular} & $42 \%$ & $\begin{array}{c}13 \\
3 \\
2 \\
6 \\
2 \\
2\end{array}$ & 1 & $\begin{array}{c}23 \\
10 \\
1 \\
3 \\
3 \\
\\
5 \\
1\end{array}$ \\
\hline 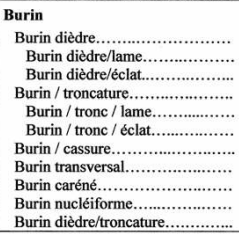 & $\begin{array}{c}\mathbf{8 1} \\
19 \\
10 \\
9 \\
36 \\
6 \\
30 \\
6 \\
4 \\
2 \\
13 \\
13 \\
1\end{array}$ & \begin{tabular}{|c|}
$19 \%$ \\
$5 \%$
\end{tabular} & $\begin{array}{l}2 \\
1 \\
1\end{array}$ & 1 & $\begin{array}{l}5 \\
2 \\
1 \\
1 \\
1 \\
1 \\
2\end{array}$ \\
\hline Grattoir-burin & 2 & $0,5 \%$ & & & \\
\hline 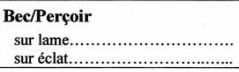 & $\begin{array}{l}56 \\
17 \\
39 \\
\end{array}$ & $13 \%$ & $\begin{array}{l}2 \\
1 \\
1 \\
\end{array}$ & & 5 \\
\hline Troneature & 62 & $15 \%$ & & 1 & 5 \\
\hline 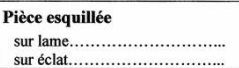 & $\begin{array}{c}30 \\
4 \\
26\end{array}$ & $7 \%$ & 2 & $\begin{array}{l}1 \\
1\end{array}$ & 5 \\
\hline Raclette & 12 & $3 \%$ & & & \\
\hline Racloir & & & & 1 & \\
\hline Lamelle à dos & 1 & $0,2 \%$ & & & \\
\hline $\begin{array}{l}\text { Lame retouchée } \\
\text { retouches continues................. } \\
\text { encoches/denticultés............... } \\
\text { retouches marginales................ }\end{array}$ & $\begin{array}{l}520 \\
132 \\
101 \\
287\end{array}$ & $\begin{array}{l}- \\
\bar{z} \\
\overline{-}\end{array}$ & $\begin{array}{c}32 \\
4 \\
10 \\
18\end{array}$ & $\begin{array}{l}9 \\
1 \\
4 \\
4\end{array}$ & $\begin{array}{c}38 \\
5 \\
21 \\
12\end{array}$ \\
\hline 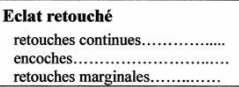 & $\begin{array}{l}267 \\
99 \\
20 \\
148\end{array}$ & $\begin{array}{l}- \\
- \\
\overline{-}\end{array}$ & $\begin{array}{c}19 \\
4 \\
11 \\
4\end{array}$ & $\begin{array}{l}3 \\
1 \\
1 \\
1\end{array}$ & $\begin{array}{c}20 \\
7 \\
11 \\
2\end{array}$ \\
\hline TOTAL OUTILS & 1205 & - & 70 & 19 & 101 \\
\hline $\begin{array}{l}\text { TOTAL OUTILS (sans lames et } \\
\text { éclats retouchés) }\end{array}$ & 418 & $\begin{array}{l}100 \\
\%\end{array}$ & 19 & 7 & 43 \\
\hline
\end{tabular}

22 Si l'on retire les lames et les éclats retouchés du décompte général, ce sont les grattoirs qui dominent à $42 \%$; ils ont été réalisés sur tous types de supports (éclat, lame), parfois épais $(>1,5 \mathrm{~cm})$; il faut remarquer la part importante qu'occupent les carénés (fig. 4 et 5). 
Figure 4 - Industrie lithique de la zone 1 (dessins de M. Jarry). Figure 4 - Lithic industry of the area 1.

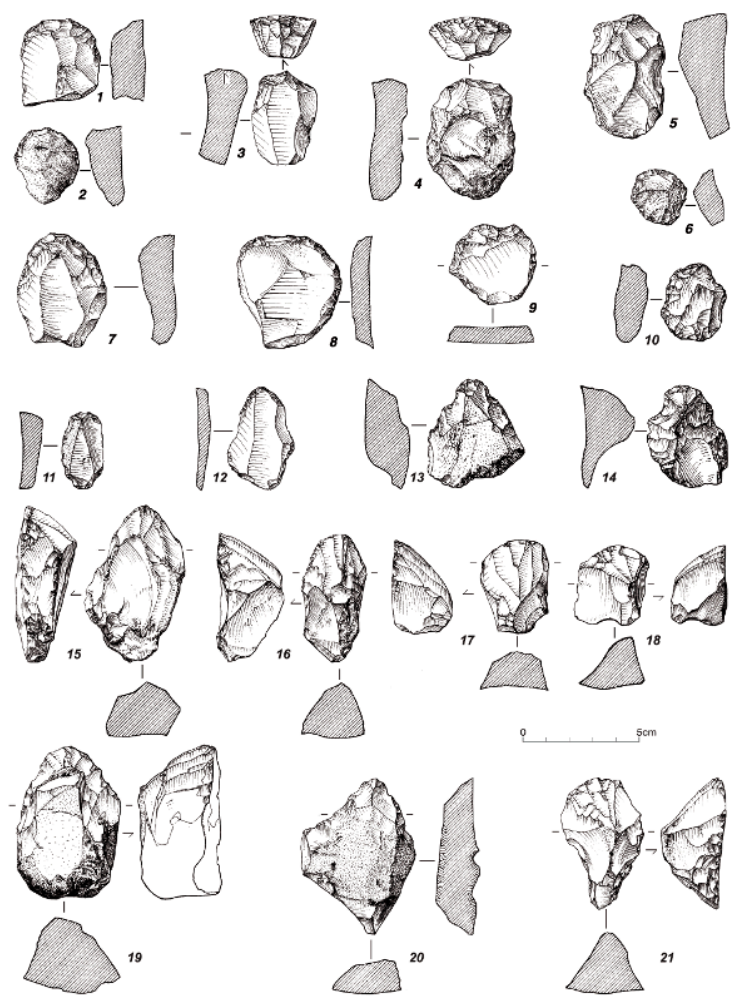

1 à 10 : grattoirs sur éclat. -11 : grattoir double. -12 à 14,20 : grattoirs à museau. -15 à 21 : grattoirs carénés. 
Figure 5 - Industrie lithique de la zone 1, Las Sablas et Coustaret (dessins de M. Jarry). Figure 5 - Lithic industry of the area 1, Las Sablas and Coustaret.
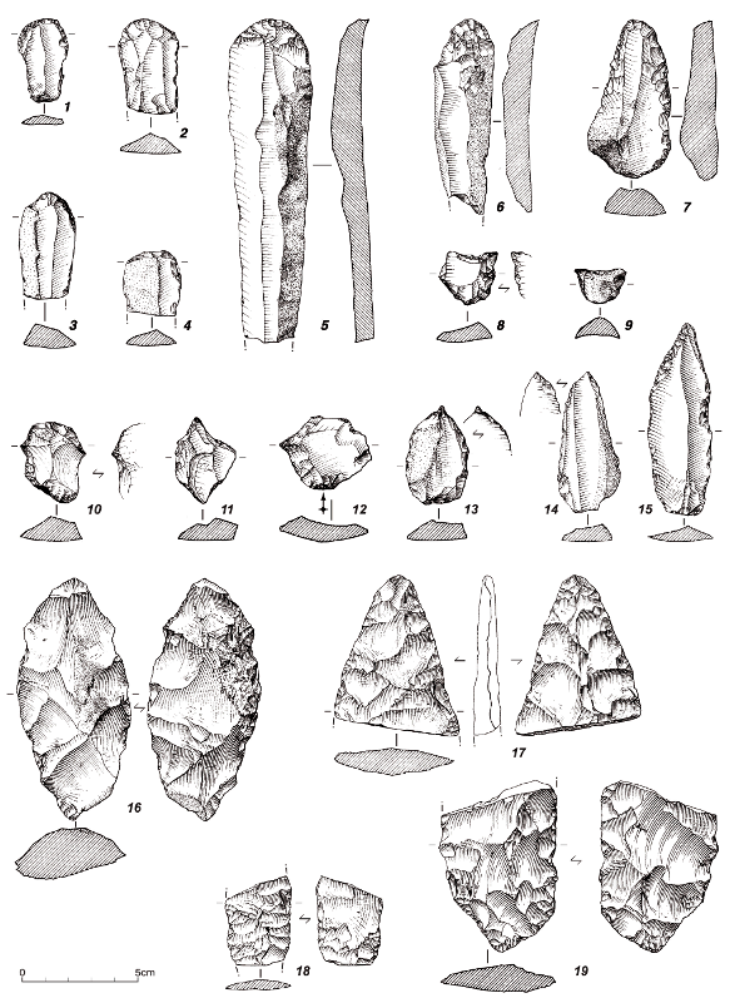

1 à 7 : grattoirs sur lame (le $n^{\circ} 5$ est sur support allochtone encore indéterminé). - 8 à 15 : becs ou perçoirs. -16 : ébauche de pièce bifaciale. -17 à 19 : pièces bifaciales solutréennes (16 à 18 : Las Sablas - 19 : Coustaret).

Les burins sont présents pour $19 \%$. Les burins sur troncatures (9\%) dépassent les burins dièdres $(5 \%)$. Leurs supports sont autant des éclats que des lames et sont souvent très épais $(2$ à $2,5 \mathrm{~cm}$ ) et notamment certains qui pourraient être aussi bien des nucléus à lamelles (fig. 6). 
Figure 6 - Industrie lithique de la zone 1 (dessins de M. Jarry). Figure 6 - Lithic industry of the area 1.

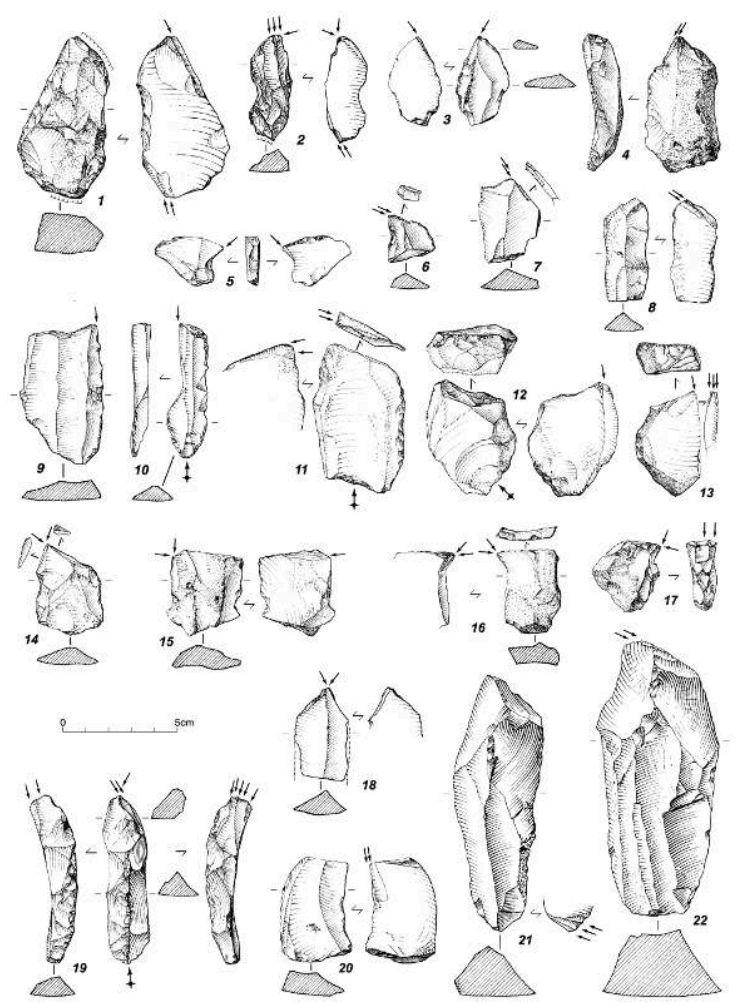

$1,3,4,5,7,9,10,12,13,17$ : burins sur troncature. $-6,16$ : burin-bec. -11 : burin transversal sur encoche. -2 : burin mixte (burin dièdre et sur troncature). $-14,17,18,19$ : burin dièdre. -15 : burin dièdre d'angle. $-8,20$ : burin sur cassure. $-21,22$ : burins dièdres sur supports robustes.

Les troncatures représentent $15 \%$ de l'ensemble. Elles ont été réalisées exclusivement sur des supports laminaires qui peuvent être des petites lames plus ou moins épaisses (fig. 7) ou des pièces robustes. 
Figure 7 - Industrie lithique de la zone 1 et de Las Vignes (dessins de M. Jarry). Figure 7 - Lithic industry of the area 1 and Las Vignes.
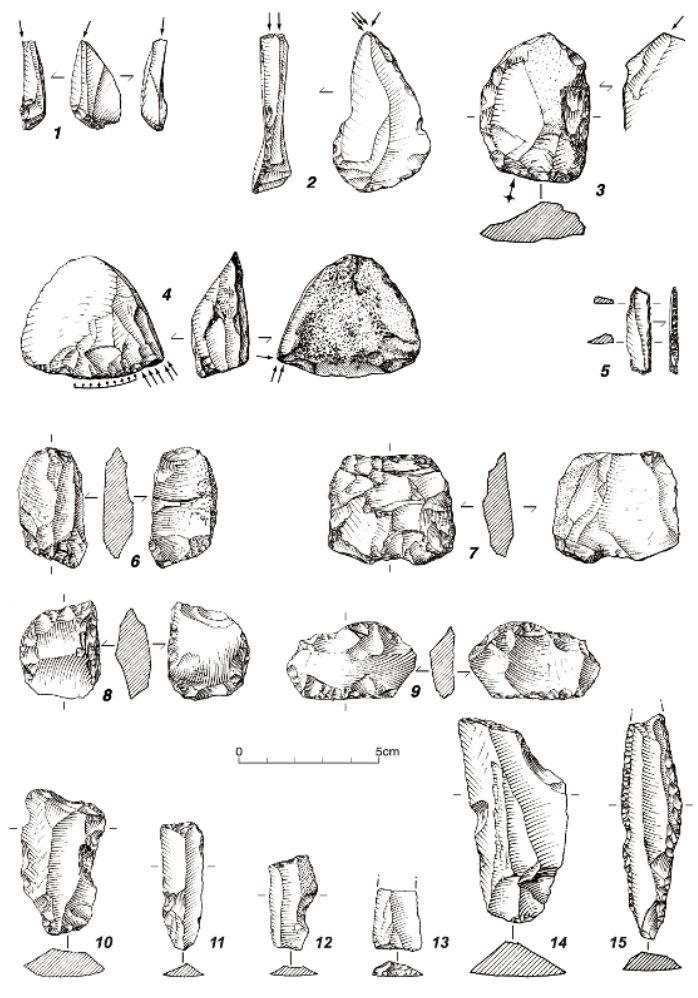

1,2 : grattoir-burin. -3 : burin sur troncature à enlèvement plan. -4 : burin nucléiforme. -5 : lamelle à dos. -6 à 9 : pièces esquillées. - 10 à 14 : lames à troncature. -15 : pointe de Châtelperron.

Les becs/perçoirs (13\%) sont majoritairement réalisés sur éclats ; leurs rostres peuvent être bien dégagés ou parfois aménagés de manière sommaire (fig. 5).

Les pièces esquillées ( $7 \%$ ), majoritairement sur éclat sont du même type que celles qu'on retrouve dans les habitats en grotte ou en abri, notamment dans les niveaux gravettiens (fig. 7). On notera enfin la présence de quelques raclettes (3\%).

En ce qui concerne les lames retouchées (fig. 8), nous les avons classées selon les trois catégories suivantes :

- lames à encoches ou/et denticulées ;

- lames à retouches marginales : elles sont souvent ponctuelles (elles n'affectent qu'une petite partie de la lame), alternes ; elles peuvent être des retouches d'utilisation (grignotage) ;

- lames à retouches continues : elles affectent une bonne partie de la lame; elles sont souvent très développées et d'une ampleur importante.

Figure 8 - Industrie lithique de la zone 1 (dessins de M. Jarry).

Figure 8 - Lithic industry of the area 1. 


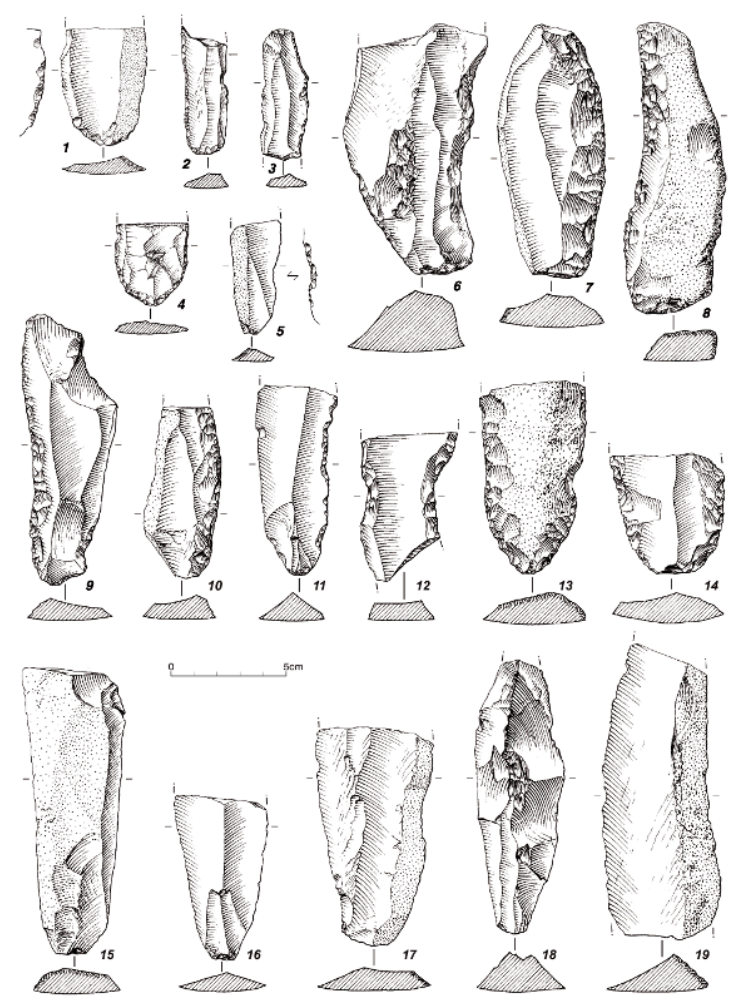

1 à 14 : lames retouchées. - 15 à 19 : lames brutes de débitage.

Nous avons adopté les mêmes critères de classement pour les éclats retouchés puisqu'on retrouve les mêmes type de retouches : seul le support a changé.

Bien que l'atelier se trouve sur le gîte de matière première, des supports en silex allochtones sont aussi présents (tabl. 3). Ils représentent $8 \%$ de tous les outils confondus. C'est le silex de Chalosse qui a été majoritairement employé (5,5\%), puis un silex dont l'origine est à rechercher dans les affleurements du flysch du piémont béarnais. Les silex des Prépyrénées restent d'emploi restreint; on notera l'utilisation du silex du Paillon. Les types d'outils principalement façonnés dans ces silex sont les grattoirs et les lames retouchées. 
Tableau 3 - Décompte des outils en silex allochtones de la zone 1. Table 3 - Allochthonous flint tools counts of the area 1.

\begin{tabular}{|c|c|c|c|c|c|c|c|}
\hline Types d'outils & $\begin{array}{l}\text { ஜ } \\
\frac{0}{0} \\
\frac{0}{0}\end{array}$ & 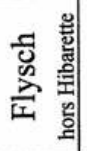 & 吾 & 总 & 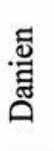 & 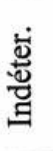 & 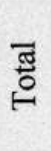 \\
\hline $\begin{array}{l}\text { Grattoir } \\
\text { Grattoir / éclat..................... } \\
\text { Grattoir / lame..................... } \\
\text { Grattoir caréné.................... }\end{array}$ & $\begin{array}{c}24 \\
17 \\
7\end{array}$ & $\begin{array}{l}8 \\
6 \\
2\end{array}$ & & $\begin{array}{l}2 \\
2\end{array}$ & $\begin{array}{l}2 \\
1 \\
1\end{array}$ & $\begin{array}{l}6 \\
4 \\
1 \\
1\end{array}$ & 42 \\
\hline $\begin{array}{l}\text { Burin } \\
\text { Burin / troncature..................... }\end{array}$ & & & $\begin{array}{l}1 \\
1\end{array}$ & & & & 1 \\
\hline Bec/Perçoir & 2 & & & & & 2 & 4 \\
\hline Troncature & 5 & & & & & 1 & 6 \\
\hline Pièce esquillée & 2 & & & & & 1 & 3 \\
\hline Raclette & 2 & & & & 1 & & 3 \\
\hline Lamelle à dos & 1 & & & & & & 1 \\
\hline $\begin{array}{l}\text { Lame retouchée } \\
\text { retouches continues................. } \\
\text { encoches/denticulées................ } \\
\text { retouches marginales............... }\end{array}$ & $\begin{array}{c}21 \\
8 \\
6 \\
7 \\
\end{array}$ & & & & & 3 & 24 \\
\hline Eclat retouché & 9 & & & & & 2 & 11 \\
\hline TOTAL OUTILS & 66 & 8 & 1 & 2 & 3 & 15 & 95 \\
\hline $\begin{array}{l}\text { TOTAL OUTILS (sans lames et } \\
\text { éclats retouchés.) }\end{array}$ & 36 & 8 & 1 & 2 & 3 & 10 & 60 \\
\hline
\end{tabular}

\section{Zones 2,3 et 4}

L'effectif des outils des zones 2 et 3 est notoirement insuffisant pour en tirer une quelconque analyse si ce n'est qu'on se situe vraisemblablement en marge de l'occupation principale. Pour la zone 4, le nombre d'outils est plus élevé (101), sans pour autant être comparable à celui de la zone 1. Cependant, on retrouve presque la même diversité typologique que dans la zone 1 et la composante aurignacoïde reste prépondérante.

\section{Quelques données d'ordre technologique}

\section{Etude des nucléus de la zone 1}

31 Nous présenterons ici une brève description des différents nucléus trouvés dans la zone 1 qui est la principale zone de concentration d'artefacts. Nous avons écarté d'emblée tous les types caractéristiques du Paléolithique moyen, c'est-à-dire tous ceux qui présentent un débitage centripète de type Discoïde ou Levallois. Le nombre de nucléus ainsi totalisé, retenu pour notre étude, est de 528. Leur répartition selon les grands types est la suivante : 124 nucléus à lames, 60 nucléus à éclats laminaires, 131 nucléus à lamelles, 213 nucléus à éclats. 
Figure 9 - Schéma diacritique des nucléus à éclats et à lames de la zone 1 (dessins de P. Foucher). Figure 9 - Diacritical sketch of flakes and blades cores of the area 1.

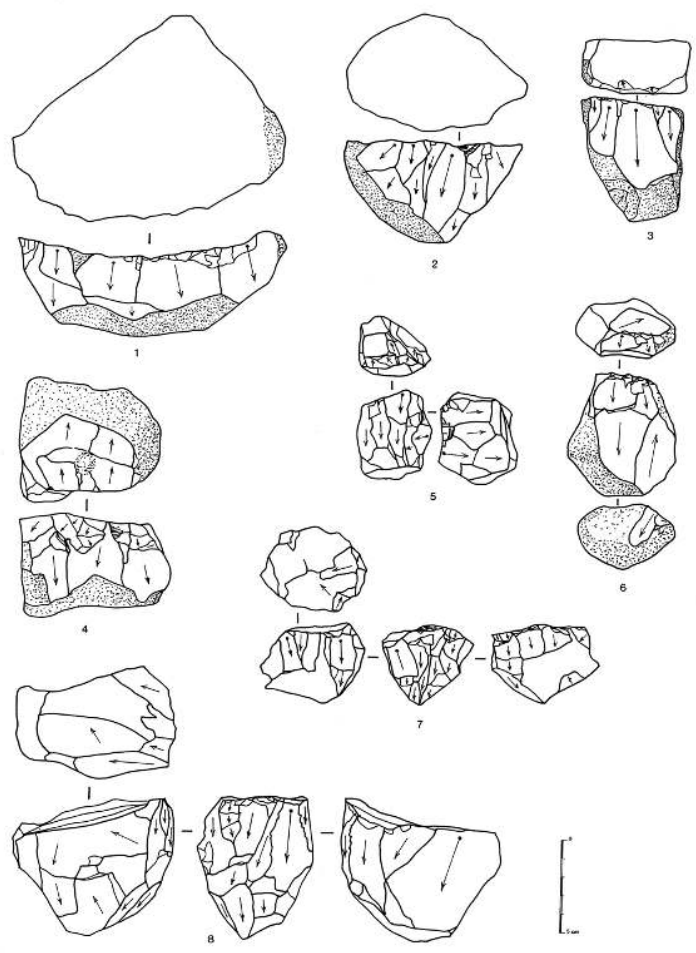

1 à 3 : nucléus à éclats à plan de frappe naturel. -4 à 6 : nucléus à plan de frappe préparé. $-7,8$ : nucléus à lames de type caréné dont la production finale se termine par des éclats laminaires. 
Figure 10 - Schéma diacritique des nucléus à lames de la zone 1 (dessins de P. Foucher). Figure 10 - Diacritical sketch of blades cores of the area 1.
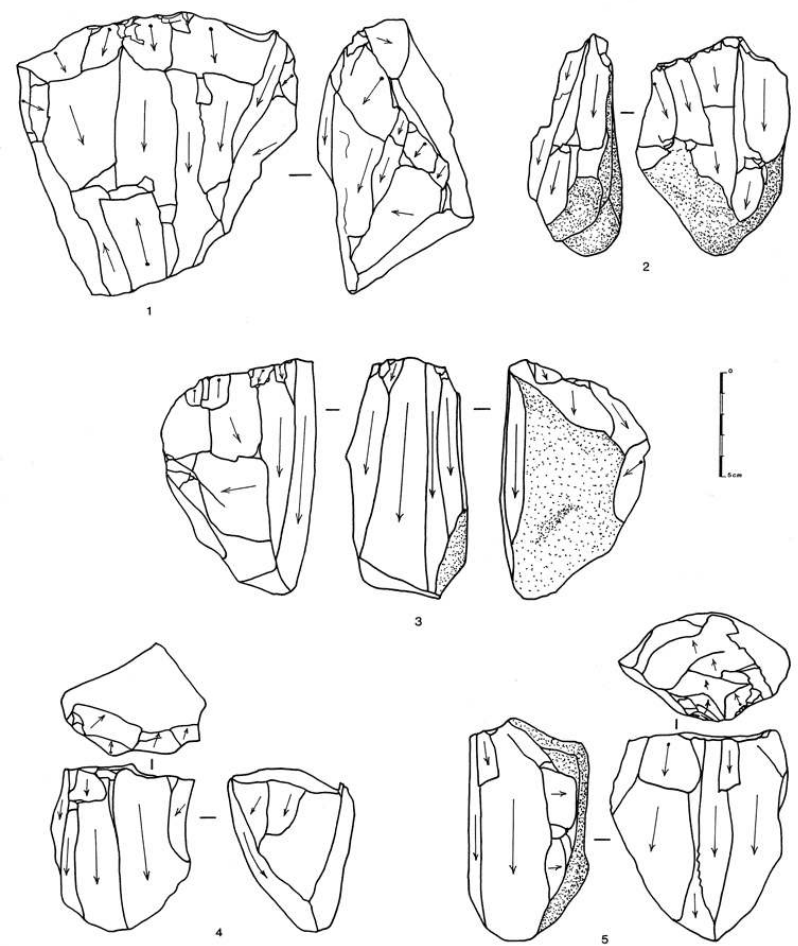

$1,3,5$ : nucléus sur rognons volumineux destinés à une production de lames larges relativement longues. -2 : nucléus à un plan de frappe, à table large et à lames courtes mais larges. 
Figure 11 - Schéma diacritique des nucléus à lames de la zone 1 (dessins de P. Foucher). Figure 11 - Diacritical sketch of blades cores of the area 1.
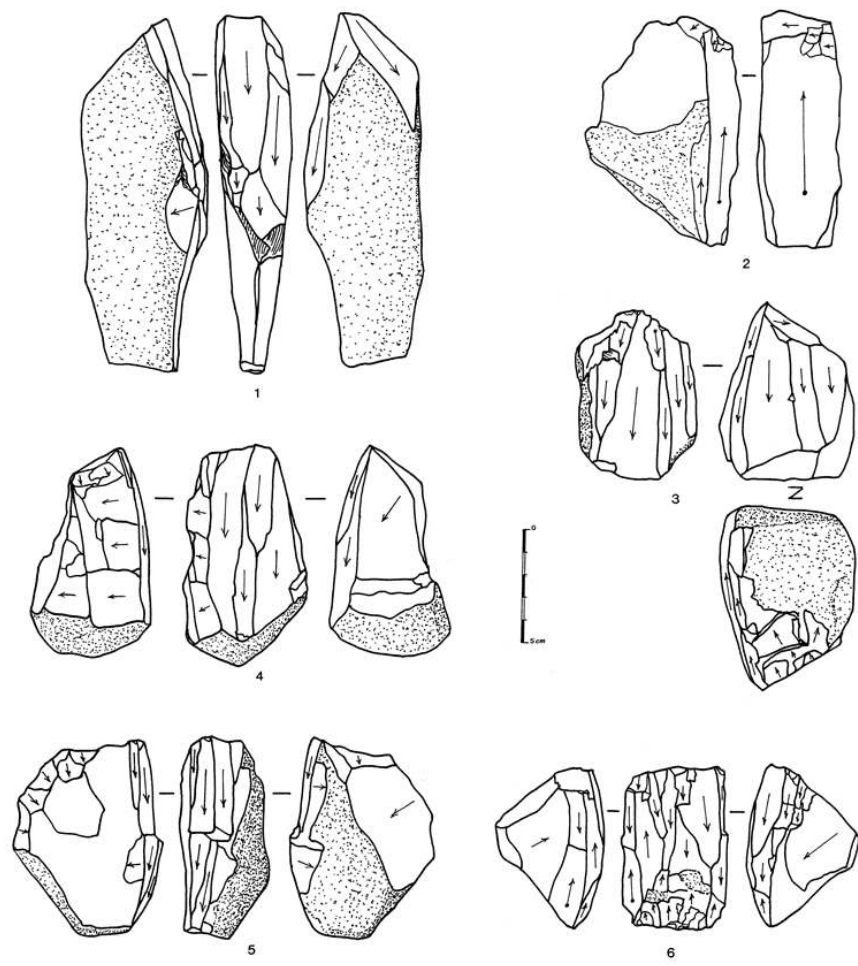

1,2 : nucléus développé sur plaquette, avec un débitage à recul frontal. - 3 : nucléus à un plan de frappe pour une production de lames courtes mais larges. -4 : nucléus prismatique à un plan de frappe. - 5 : nucléus réalisé à partir d'un gros éclat. 
Figure 12 - Schéma diacritique des nucléus à lamelles de la zone 1 (dessins de P. Foucher). Figure 12 - Diacritical sketch of bladelets cores of the area 1.

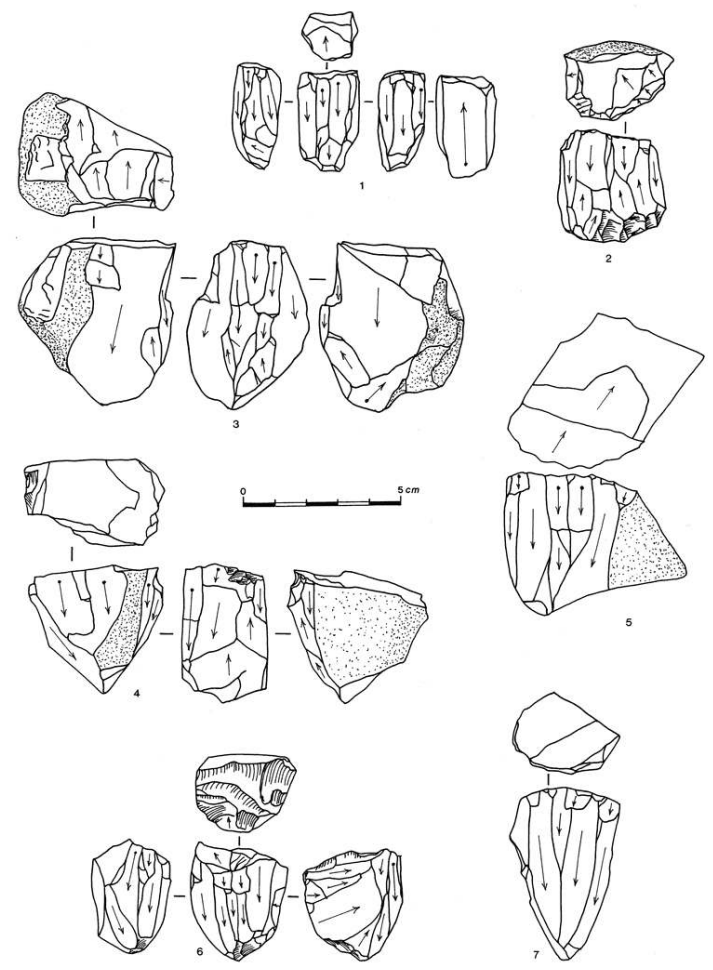

1 : nucléus prismatique à lamelles à un plan de frappe préférentiel. $-2:$ nucléus à lamelles à deux plans de frappe et à dos naturel cortical. - 3 : nucléus à lamelles à débitage frontal et à dos naturel. 4 : nucléus à lamelles à débitage frontal sur plaquette (deux plans de frappe). - 5 : nucléus à lamelles à un plan de frappe sur plaquette. $-6,7$ : nucléus à lamelles à débitage convergent.

- Blocs testés (18)

- Préformes (12)

- Dans le cas des nucléus à éclats (213), nous avons gardé pour étude des types qui ne rentraient pas a priori dans ceux du Paléolithique moyen ou ancien; il se peut que certains exemplaires n'appartiennent pas au Paléolithique supérieur ou que nous en ayons écarté d'autres qui devaient l'être :

- nucléus à éclats atypiques (20) dont le débitage est désordonné et la production atteint trois ou quatre éclats ; leurs formes sont plus ou moins polyédriques ; l'arête des négatifs d'éclats est d'environ 7 à $5 \mathrm{~cm}$;

- nucléus à éclats (59), réalisés à partir d'un plan de frappe naturel (négatif d'un éclat de gel, plan de clivage du rognon, etc.) ; les produits recherchés sont des éclats obtenus dans la zone sous-corticale du rognon (fig. $9: 1$ à 3);

- nucléus à éclats (134), réalisés à partir d'un plan de frappe préparé (fig. $9: 4$ à 6) ; leurs formes sont plus ou moins polyédriques ; les techniques de débitage au percuteur dur sont majoritairement employées ; les différentes classes de taille (longueurs des arêtes des négatifs d'éclats) et leurs effectifs sont les suivants :

- arêtes de 2 à $3 \mathrm{~cm}=40$

- arêtes de 3 à $4 \mathrm{~cm}=55$

- arêtes de 5 à $6 \mathrm{~cm}=33$

- arêtes de 7 à $8 \mathrm{~cm}=6$ 
- Pour les nucléus à lames (124), nous avons distingué les types suivants :

- nucléus à un plan de frappe, à table large qui a souvent gagné sur le flanc du nucléus (18); la production recherchée est celle de lames courtes mais larges (entre 5-6 cm et 6-7 cm) - fig. $10: 2$ et 4 ; fig. $11: 3$;

- nucléus à un plan de frappe, avec un débitage tournant sur bloc épais qui dégage un front (19) ; la forme qui en résulte se rapproche des grattoirs carénés, mais pour une production de lames (fig. $9: 7$ et 8 );

- nucléus développés sur plaquette, avec un débitage à recul frontal dans le volume du bloc (21) - fig. $11: 1$ et 2 ;

- nucléus prismatiques à un plan de frappe (26) - fig. 11:4 - leurs dos sont souvent naturels (plan de clivage, plage corticale) ou aménagés par deux enlèvements croisés qui déterminent plus un "pincement" qu'une crête ;

- nucléus à deux plans de frappe (21), pour une production de lames droites et courtes (fig. $11: 6)$;

- nucléus réalisés à partir de gros éclats (5) - fig. 11:5 - ces types de nucléus se retrouvent souvent dans les gîtes/ateliers des Prépyrénées; ce mode de production, qui consiste à prélever un gros éclat destiné à être emporté vers les habitats et devenir un nucléus à lamelles, est en partie déterminé par la qualité des matières premières qui sont souvent très hétérogènes (notamment le "Bleu" pyrénéen) : il est plus aisé de rapporter un fragment de matière qui pourra être débité que de s'embarrasser d'un volumineux rognon qui pourrait s'avérer être impropre à la taille (Simonnet 1999) ;

- nucléus sur rognons volumineux pour une production de lames larges relativement longue (10 cm de long et 3 à $4 \mathrm{~cm}$ de large) - fig. $10: 1,3,5$;

- un nucléus qui laisse penser à un débitage au punch.

- Pour les nucléus à lamelles (131), nous avons distingué les types suivants :

- nucléus à éclats lamellaires (14) ; le débitage est souvent maladroit ; il est le pendant de celui que l'on retrouve sur des nucléus plus gros ; ils semblent être l'exemple de pièces d'apprentissage ;

- nucléus à lamelles à un plan de frappe, de section plus ou moins parallélépipédique, à débitage à recul frontal (29); il rentre dans ce type les nucléus à lamelles sur fragment de petite plaquette (fig. $12: 3$ et 4 );

- nucléus à lamelles à un plan de frappe et à débitage convergent (10); il rentre dans ce type les nucléus à lamelles pyramidaux (fig. $12: 6$ et 7);

- nucléus à lamelles à un plan de frappe et à débitage qui se développe sur les flancs du nucléus (10) - fig. $12: 5$;

- nucléus à lamelles, de type prismatique à débitage tournant (12) - fig. $12: 1$;

- nucléus à lamelles de type caréné (16); la différence avec les grattoirs carénés est malaisée à établir ; on classera plutôt certaines pièces comme nucléus celles qui présentent un front (ou une table) moins régulière ; ce type de pièce pourrait alimenter le débat sur la nature et fonction des grattoirs carénés que certains considèrent comme des nucléus (Bon 2000), de même que celles de la figure 9 (7-8);

- nucléus à lamelles à deux plans de frappe (31) ; souvent la table est large et le débitage des lamelles s'est effectué à partir des deux plans de frappe ; dans d'autres cas, le deuxième ne semble avoir servi qu'à la réfection de la table (fig. $12: 2$ );

- nucléus à lamelles divers (8).

- Il faut souligner la présence de nombreux percuteurs (30) :

- sur galets de quartzite, granite et roche métamorphique (12); 
- sur petits rognons de silex de Montgaillard/Hibarette (16);

- sur d'anciens petits nucléus en silex de Chalosse (2).

\section{Attribution typo/techno-chronologique}

Comme nous l'avons déjà indiqué, l'industrie récoltée à Hibarette témoigne de nombreuses occupations à des périodes très diverses de la Préhistoire, ce qui limite la portée d'une étude typo-technologique très poussée. Néanmoins, certains éléments typo-technologiques repérés permettent de mieux préciser quelques champs chronologiques pour le Paléolithique supérieur.

Les ateliers semblent avoir fonctionné plus particulièrement à l'Aurignacien si l'on tient compte du nombre relativement important des grattoirs carénés, des grattoirs sur lames ou éclats robustes ainsi que des lames à retouches aurignaciennes trouvés sur place. A cette période, correspondraient les nombreux nucléus à lames larges dont une partie des supports a servi directement dans l'outillage aurignacien du site. Cependant, la part principale de la production laminaire a dû être emportée vers les habitats pyrénéens : nous n'avons retrouvé que 155 lames brutes et 12 lamelles sur le gisement, ce qui est une quantité dérisoire comparée à la masse de matière qui a été débitée.

40 La présence solutréenne est à signaler hors des 4 zones précédemment définies, par une pièce foliacée au lieu-dit Las Sablas et par une concentration d'industrie solutréenne caractéristique au lieu-dit Coustaret $^{3}$ (fig. $5: 16$ à 19); cette dernière semble être homogène et elle fera l'objet d'une étude séparée.

L'autre occupation importante est celle des Magdaléniens. A la phase ancienne de cette culture peuvent correspondre les raclettes, certains perçoirs sur éclat et les pièces esquillées, bien que ces dernières puissent être également une production aurignacienne ou gravettienne, voire azilienne. L'existence d'une chaîne opératoire axée sur la production d'éclats conforterait l'attribution au Magdalénien ancien. Pour la phase récente, il n'existe dans l'ensemble étudié aucune pièce caractéristique pouvant s'y rattacher; cependant, de nombreux nucléus à lames étroites et surtout à lamelles évoquent particulièrement le mode de débitage du Magdalénien moyensupérieur (fig. 11:1, 5, 6 ; fig. 12:6,7) ; nous retrouvons le même type de nucléus, tant au niveau des formes que de la matière première employée, notamment des plaquettes de silex de Montgaillard/Hibarette, dans le gisement magdalénien de la grotte de Labastide, distant de trente kilomètres (Simonnet 1991).

D'autres indices permettent d'évoquer quelques passages à d'autres périodes, spécialement le Châtelperronien ; il existe une pointe de Châtelperron (Las Vignes) qui entre dans la variabilité des formes du type (fig. $7: 15$ ) : la pointe est cassée, le dos présente des retouches semi-abruptes; celles de la corde sont continues, ce qui pourrait la distinguer des pièces standard. Le support de la pièce est en silex de Chalosse et elle a dû être abandonnée en raison de sa cassure. Les rapprochements immédiats sont à faire avec le Châtelperronien de la grotte de Gatzarria (Laplace 1966) à l'ouest, ainsi qu'avec le site de plein air des Tambourets (Méroc 1963) à l'est. L'Epipaléolithique n'est guère décelable puisque aucune pièce typique ne se retrouve dans l'industrie analysée, mais une étude récente des niveaux épipaléolithiques de la grotte de Troubat indique l'utilisation du silex de Montgaillard/Hibarette en proportion importante dans l'outillage (Lacombe 1998, 1999); certains petits nucléus à éclats laminaires pourraient être le résultat d'une taille à cette période (fig. $9: 2,3,6$ ). 
De même, par l'existence de silex Montgaillard/Hibarette dans les niveaux gravettiens de Gargas et d'Enlène EDG (Foucher, San Juan, à paraître), on peut inférer que les Gravettiens se sont arrêtés sur le gîte et il est possible que certains nucléus puissent se rattacher à des modalités de débitage de cette culture. Enfin, il existe sur le gisement des pointes de flèches et des haches polies qui révèlent des fréquentations au Néolithique; de nombreux grattoirs, notamment en silex de Chalosse, pourraient se rapporter à cette époque (grattoirs sur éclat ou éclat laminaire avec un bulbe épais et un talon lisse large).

\section{La place et l'importance du gîte d'Hibardette dans l'économie des matières premières du monde pyrénéen}

43 Le gîte/atelier d'Hibarette se présente comme un site étendu, à exploitation extensive, couvrant plusieurs hectares (un peu plus de $1 \mathrm{~km} \_$) dont une bonne partie n'est pas accessible actuellement ou a été malheureusement détruite (à l'emplacement de la déchetterie). Il est relativement isolé par rapport aux gîtes pyrénéens les plus proches comme ceux d'Arudy (40 km), de Lespugue-Montmaurin (40 km), de Salies-du-Béarn (60 $\mathrm{km})$, du Dôme d'Aurignac, de Courensan dans le Gers $(76 \mathrm{~km})$ ou de Chalosse $(85 \mathrm{~km})$.

La genèse de la matière siliceuse se place dans les formations du flysch (Crétacé supérieur). A la suite d'un démantèlement de cette formation et d'un transport, le silex s'est retrouvé au sein des poudingues de Palassou. Mais les affleurements de silex accessibles aux Paléolithiques se situaient dans les colluvions remaniant les poudingues à la suite des phénomènes érosifs, fréquents en contexte climatique périglaciaire et en piémont montagnard.

Les affleurements sont très riches en matière première qui possède une bonne aptitude à la taille, et livrent des rognons de toutes dimensions, permettant des débitages de lames de plus de $15 \mathrm{~cm}$ de long, et de toutes formes: ceux en plaquettes ont été particulièrement employés à cause de leur forme parallélépipédique qui ne nécessite quasiment aucune préparation avant le plein débitage de lames.

L'outillage trouvé sur le site est numériquement important et très diversifié dans ses composantes typologiques; il se rapproche de celui qu'on rencontre dans les habitats classiques en grotte ou abri (à faciès domestique dominant).

Le site a été fréquenté sur la longue durée, du Paléolithique moyen au Néolithique. Les ateliers de taille du Paléolithique supérieur semblent se rapporter surtout à l'Aurignacien, au Solutréen et au Magdalénien, avec quelques indices pour le Châtelperronien, le Gravettien et l'Epipaléolithique.

On peut le rapprocher des gîtes/ateliers du Pla (Mas d'Azil, Ariège), de Jean Nougué (Fabas, Ariège) ou de Bouzin village (Haute-Garonne) ${ }^{4}$ qui présentent les mêmes caractéristiques : longue durée de fréquentation et diversité de l'outillage associé (à la nuance près que l'outillage de Hibarette est numériquement plus important). En revanche, il se démarque de la myriade d'ateliers des Petites Pyrénées qui se répartissent en continu sur un front de $70 \mathrm{~km}$ et où l'on retrouve une majorité de déchets de débitage. 
49 La situation qu'occupe Hibarette dans l'espace paléolithique et le réseau d'habitats en grotte (ou abri) n'est pas la même que celle qu'on trouve dans les Petites Pyrénées. Les premiers karsts habités sont Lourdes $(12 \mathrm{~km})$, Aurensan $(12 \mathrm{~km})$, Espèche $(20 \mathrm{~km})$, Labastide $(28 \mathrm{~km})$, tandis que, dans les Petites Pyrénées, le maillage des grottes et abris se surimpose à celui des gîtes.

50 Ces contextes différents ont dû certainement avoir des incidences sur les modes de fréquentation des gîtes. Pour se fournir en silex d'Hibarette, il fallait que les paléolithiques s'y rendent pour au moins une journée complète ou bien s'y arrêtent au cours de leurs déplacements épisodiques; ce gîte/atelier constituerait un lieu de production laminaire associé à un habitat que l'on pressent plutôt de courte durée (quelques jours). Quant à la majorité des sites des Petites Pyrénées - à l'exception des grands gîtes/ateliers du Pla, Jean Nougué ou Bouzin village qui auraient le même statut que celui d'Hibarette - ils semblent n'avoir été exploités que de manière occasionnelle par des paléolithiques dont les habitats devaient être proches, pour l'obtention de quelques supports. Les gîtes sont sur place, comme la faune ; on peut s'y approvisionner au fur et à mesure des besoins sans que cela nécessite la mise sur pied d'une expédition spécialisée. Si on a besoin d'un bloc, on va le chercher (à l'exemple des occupants du Mas d'Azil, de Tarté, de Marsoulas, de Roquecourbère ou de Montmaurin-Lespugue).

51 L'analyse de l'éventail des silex allochtones présents sur le site pourrait aussi donner quelques pistes d'interprétation sur les caractéristiques des séjours paléolithiques. A Hibarette, le silex provenant de Chalosse est majoritaire : 70 \% des silex allochtones. La composante nord-aquitaine (silex du Bergeracois, gris ou noir du Sénonien périgourdin) est absente, alors que celle-ci se retrouve systématiquement dans les cortèges lithiques des habitats des Pyrénées centrales. Cette donnée pourrait aller dans le sens d'une occupation de courte durée du site d'Hibarette : en effet, si le séjour s'était prolongé, les paléolithiques auraient pu abandonner leurs outils de voyage en silex périgourdins comme c'est le cas en grotte ou sous abri (la logique commande que plus on reste longtemps dans un endroit, plus on est amené à abandonner des ustensiles que l'on a usés). L'hypothèse que l'on peut retenir serait une occupation de courte durée par quelques personnes, détachées du groupe principal dont l'habitat prolongé est ailleurs, venues spécialement sur le gîte pour des opérations de taille qui ont pu leur demander quelques jours (prospection et taille). L'apport de matériaux issus des sites de Chalosse suggère que leur dernière provenance connue est certainement à rechercher à l'ouest des Pyrénées.

52 Les hypothèses énoncées doivent être relativisées à l'aune du contexte d'étude qui est celui d'une prospection, certes fine et systématique, mais qui possède ses limites : nous sommes loin d'avoir collecté toute l'industrie débitée sur le gisement et il reste très difficile d'appréhender la fréquence des passages des paléolithiques. Elles doivent donc être considérées comme les "scénarios" les plus probables (de même pour les campements en retrait par rapport au piémont occupés par des groupes plus importants et dans la mesure où on a une idée de l'importance numérique de ces groupes).

53 Les études sur l'origine des matières siliceuses dans les habitats pyrénéens sont suffisamment bien avancées pour avoir une bonne idée de la diffusion de ces différents types de matériau (Simonnet 1973, 1981, 1999 ; Simonnet, Simonnet 1991; Chalard et alii 1995 ; Lacombe 1998 ; Normand 1986 ; Bon 2000 ; Briois 2000 ; Foucher, San Juan 2000). Dans le cas du silex de Montgaillard/Hibarette, on le retrouve dans presque tous 
les principaux sites des Pyrénées centrales se rapportant au Paléolithique supérieur et dans des proportions qui varient en fonction de l'éloignement du site par rapport au gîte. La figure 13 synthétise les données actuellement connues sur la circulation de cette matière siliceuse.

Figure 13 - Localisation des sites du Paléolithique supérieur ayant livré des supports en silex de Montgaillard/Hibarette (carte M. Jarry et P. Foucher).

Figure 13 - Location of the Upper Palaeolithic sites that provided Montgaillard/Hibarette flint blanks.

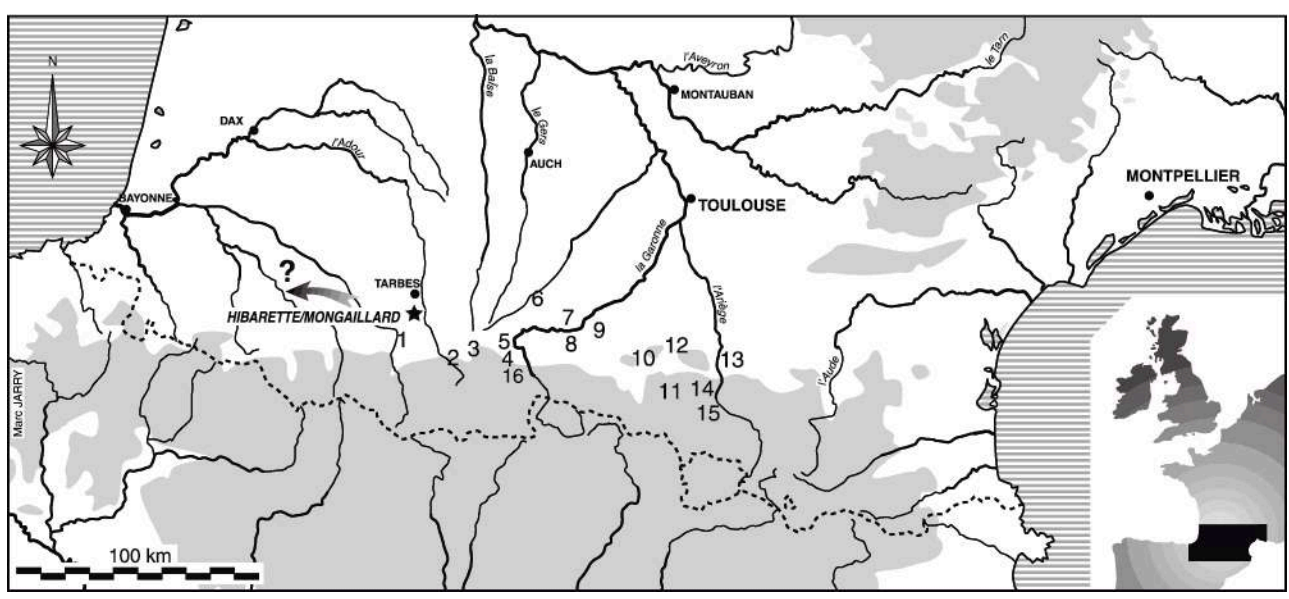

1 : Les Espélugues (Magd.). 2 : Labastide (Magd.). 3 : Lortet (Magd.). 4 : Gargas (Grav.). 5 : Gourdan (Magd.). 6 : Lespugue (Sol. et Magd.). 7 : La Tourasse (Magd.). 8 : Tarté (Aurig.). 9 : Roquecourbère (Sol.). 10 : Enlène (Grav.). 11 : Massat (Magd.). 12 : Mas d'Azil (Magd.). 13 : Tuto de Camalhot (Grav.). 14: Rhodes II (Magd.). 15: La Vache (Magd.). 16: Troubat (Magd.)

54 Jusqu'à présent, le silex de Montgaillard-Hibarette n'a pu être mis en évidence que dans les sites localisés à l'est du gîte/atelier (à l'exception des Espélugues à Lourdes). Au sein des habitats qui se trouvent dans un rayon de $60 \mathrm{~km}$, comme Labastide, Gargas, Troubat, il occupe une place importante dans l'industrie lithique : par exemple plus de $20 \%$ des roches débitées dans le Magdalénien supérieur de Troubat et $16 \%$ dans l'Azilien (Lacombe 1999). Au-delà, comme par exemple dans le Solutréen de Roquecourbère, des Harpons et le Gravettien d'Enlène ou de La Tuto de Camalhot, il est présent dans l'outillage à 1 ou $2 \%$ (Foucher, San Juan 2000 et à paraître). Les deux derniers sites gravettiens se situent respectivement à 90 et $125 \mathrm{~km}$ de la source d'approvisionnement.

Il faut citer plus particulièrement le cas de la grotte de Labastide qui a l'avantage de réunir plusieurs niveaux d'informations qui, si on les recoupe, permettent de proposer un sens de diffusion culturelle dans le monde magdalénien à partir de faits archéologiques précis (Simonnet, Simonnet 1991). L'outillage des Magdaléniens qui ont séjourné dans la grotte est composé uniquement de silex originaires de l'ouest ou du nord (Silex d'Hibarette, de Chalosse ou du Périgord). Les coquillages marins percés corroborent cet axe de déplacement nord-sud ou ouest-est. Si au cours de leur périple, ils étaient passés par les Petites Pyrénées, ils auraient immanquablement ramassé des supports de cette région; ce qui semble être la règle dans les habitats des Pré-Pyrénées et des vallées intérieures lorsqu'on atteint le méridien des gîtes prépyrénéens dans un déplacement vers l'est, au pied de la chaîne. Or, jusqu'à présent aucun support de provenance orientale n'a pu être détecté. Par ailleurs, dans le corpus des objets mobiliers découverts dans la grotte, se détache un des rares exemplaires de "propulseur au faon"; il en existe un en tous points comparables à Arudy, ainsi qu'au 
Mas d'Azil et à Bédeilhac, avec pour ces derniers une iconographie certainement plus complète (représentation supplémentaire d'un oiseau). Nous avons, d'une part, les données de circulation des matières premières qui indiquent un déplacement unilatéral des Magdaléniens de l'ouest vers l'est (dans le cas où les Magdaléniens venaient de Dordogne, ils devaient passer forcément par la Chalosse pour s'y approvisionner en silex); d'autre part, des objets mobiliers confectionnés presque à l'identique dans des sites qui jalonnent les Pyrénées occidentales et centrales. Il serait étonnant, sauf à envisager des hypothèses qui répondraient à des épisodes évènementiels qui nous échapperont toujours, que le thème iconographique du Faon appliqué aux propulseurs n'ait pas été véhiculé par les mêmes personnes qui se déplaçaient de l'ouest vers l'est et dont une des provenances assurées était la Chalosse.

En confrontant ainsi industrie lithique et art mobilier, on perçoit un courant d'influence et de diffusion culturelle au Magdalénien moyen le long des Pyrénées, de sa partie occidentale vers celle orientale.

Au fur et à mesure de l'avancement des connaissances sur les gîtes à silex des Pyrénées, le site de Hibarette apparaît comme un des principaux gîtes à silex des Pyrénées centrales. Il fait partie d'un ensemble géomorphologique, corrélatif aux affleurements du flysch qui recèlent d'autres potentialités en ressources siliceuses; mais le silex de Montgaillard possède suffisamment de caractères intrinsèques qui l'individualisent bien et en font un bon marqueur. Jusqu'à présent, sa diffusion semble n'avoir pris qu'une direction exclusivement orientale et a atteint une distance de $150 \mathrm{~km}$. Cependant, des études plus systématiques à venir pourraient réserver quelques surprises : il est du domaine du possible que des supports en silex de Montgaillard/ Hibarette puissent être reconnus (si on le recherche) dans des sites éloignés comme dans les Cantabres, l'Aquitaine ou le Languedoc-Roussillon.

\section{BIBLIOGRAPHIE}

BON F.,2000 - La question de l'unité technique et économique de l'Aurignacien : réflexions sur la variabilité des industries lithiques à partir de l'étude comparée de trois sites des Pyrénées françaises (La Tuto de Camalhot, Régismont-le-Haut et Brassempouy), Thèse de Doctorat de Préhistoire de l'Université de Paris I Panthéon-Sorbonne, 425 p., 81 fig., 23 tabl., 49 pl.

BON F., CHAUVAUD D., DARTIGUEPEYROU S., GARDERE Ph., MENSAN R.,1996 - La caractérisation du silex de Chalosse, Antiquités nationales, 28, p. 33-38, 3 fig.

BON F., SIMONNET R., VEZIAN J., (en préparation) - L'équipement lithique des Aurignaciens à La Tuto de Camalhot (Saint-Jean-de-Verges, Ariège), in : actes du colloque CTHS de Toulouse, avril 2001. BRIOIS F.,2000 - Lithothèque des matières premières siliceuses, Bilan scientifique 1999 du Service Régional de l'Archéologie de Midi-Pyrénées, Ministère de la Culture et de la Communication, p. 224-225. 
CHALARD P., BRIOIS F., LACOMBE S., SERVELLE Ch.,1995 - Lithothèque régionale, Bilan scientifique 1994 du Service Régional de l'Archéologie de Midi-Pyrénées, Ministère de la Culture et de la Communication, p. 228-229.

CLOTTES J., 1976 - Les civilisations du Paléolithique supérieur dans les Pyrénées, in : LUMLEY H. de (dir.), La Préhistoire française, vol 1, Paris, CNRS, p. 1212-1231.

CLOTTES J., 1985 - Hibarette et Saint-Martin, Gallia Préhistoire : informations archéologiques de la circonscription Midi-Pyrénées, 28, 2, p. 363-364.

CLOTTES J., 1989 - Hibarette, Gallia Préhistoire : informations archéologiques de la circonscription MidiPyrénées, 32, 1, p. 144 et 171.

CLOTTES J., SIMONNET R., 1979 - Le Paléolithique final dans le bassin de Tarascon-sur-Ariège d'après les gisements des Eglises (Ussat) et de Rhodes II (Arignac), in : La fin des Temps glaciaires en Europe. Chronostratigraphie et écologie des cultures du Paléolithique final, Colloques internationaux du CNRS, Bordeaux 1977, p. 647-659, 7 fig.

COLLECTIF, 1972 - Carte géologique de Tarbes au 1/50 000e. Institut Géographique National.

FOUCHER P., SAN JUAN C.,1998 - Le complexe gravettien / solutréen des Pyrénées centrales : prospection thématique, Bilan scientifique 1997 du Service Régional de l'Archéologie de Midi-Pyrénées, Ministère de la Culture et de la Communication, 1998, p. 232.

FOUCHER P., SAN JUAN C.,1999 - Le complexe gravettien / solutréen des Pyrénées centrales : prospection thématique, Bilan scientifique 1998 du Service Régional de l'Archéologie de Midi-Pyrénées, Ministère de la Culture et de la Communication, p. 234.

FOUCHER P., SAN JUAN C.,2000a - La grotte de Roquecourbère (Betchat, Ariège) : ses industries lithiques solutréennes et la révision critique de son art pariétal, Bulletin de la Société préhistorique française, t. 97, $n^{\circ} 2$, p. 199-210, 9 fig.

FOUCHER P., SAN JUAN C.,2000b - Le complexe gravettien / solutréen des Pyrénées centrales : prospection thématique, Bilan scientifique 1999 du Service Régional de l'Archéologie de Midi-Pyrénées, Ministère de la Culture et de la Communication, p. 221.

FOUCHER P., SAN JUAN C.,à paraître - La circulation des matières siliceuses dans le Gravettien pyrénéen, in : actes du colloque CTHS de Toulouse, avril 2001.

FOUCHER P., SAN JUAN C., MARTIN H., 2000 - Le site gravettien de La Carane-3 (Foix, Ariège), Bulletin de la Société préhistorique Ariège-Pyrénées, t. LIV, 1999, p. 15-42, 16 fig.

FROSSARD E. et Ch., 1880 - Etudes sur une grotte renfermant des restes humains de l'époque préhistorique découverte à Bagnères-de-Bigorre le 4 mai 1869, Bulletin de la Société Ramond, $2^{\mathrm{e}}$ édition augmentée de 1880 .

JARRY M.,1992 - Occupation paléolithique du plateau d'Hibarette (Hautes-Pyrénées), Université de Toulouse-le-Mirail, 187 p., 42 fig. (Mémoire de maîtrise).

LACOMBE S.,1998 - Préhistoire des groupes culturels au Tardiglaciaire dans les Pyrénées centrales. Apports de la technologie lithique. Thèse de doctorat, Université de Toulouse-le-Mirail, 385 p. 100 fig.

LACOMBE S.,1999 - Stratégies d'approvisionnement en silex au Tardiglaciaire. L'exemple des Pyrénées centrales françaises, Préhistoire ariégeoise, Bull. de la Société préhistorique Ariège-Pyrénées, 1998, t. LIII, p. 223-266, 4 fig.

LAPLACE G., 1966 - Les niveaux castelperroniens, protoaurignaciens et aurignaciens de la grotte Gatzarria à Suhare en Pays Basque, Quartär, 17, p. 117-140, 4 fig., 5 tabl. 
MEROC L.,1947 - Le silex dans le bassin sous-pyrénéen de la Garonne et son emploi par l'homme préhistorique, Bulletin de la Société archéologique du Midi de la France, 3e série, tome V, p. 234-250.

MEROC L.,1953 - La conquête des Pyrénées par l'Homme, Premier Congrès International de Spéléologie, Paris, t. IV, section 5, p. 33-51.

MEROC L.,1963 - L'Aurignacien et le Périgordien dans les Pyrénées françaises et dans leur avantpays, in : Aurignac et l'Aurignacien : centenaire des fouilles d'Edouard Lartet, p. 63-74, 3 fig. (nº spécial du Bulletin de la Société méridionale de Spéléologie et de Préhistoire, t. VI-IX, 1956-1959).

NORMAND Ch.,1986 - Inventaire des gîtes à silex de la Chalosse (1984-1985), Bulletin de la Société de Borda, 402, p. 133-140, 1 fig.

SIMONNET G., L et R., 1990 - Art mobilier et art pariétal à Labastide, in : L'art des objets au Paléolithique. L'art mobilier et son contexte (tome 1), Actes du premier colloque international sur l'art mobilier paléolithique, Foix-Mas d'Azil, nov. 1987, p. 173-187, 8 fig.

SIMONNET G., L et R., 1991 - Le propulseur au faon de Labastide (Hautes-Pyrénées), Préhistoire ariégeoise, Bull. de la Société préhistorique Ariège-Pyrénées, t. XLVI, p. 133-143, 4 fig.

SIMONNET R., 1973 - Le Paléolithique supérieur entre l'Hers et la Garonne, in : Préhistoire et Protohistoire des Pyrénées françaises, Musée pyrénéen, Château de Lourdes, p. 39-44.

SIMONNET R., 1981 - Carte des gîtes à silex des Pré-Pyrénées, in : La Préhistoire en Quercy dans le contexte de Midi-Pyrénées, Congrès Préhistorique de France, Montauban-Cahors 1979, Société Préhistorique Française, p. 308-323, 2 fig.

SIMONNET R., 1982 - Grandes lames de silex dans le Paléolithique supérieur des Pyrénées centrales, Préhistoire ariègeoise, Bull. de la Société préhistorique Ariège-Pyrénées , t. 37, p. 61-106, 12 fig., 1 tabl.

SIMONNET R., 1985 - Le silex du Magdalénien final de la grotte des Eglises dans le bassin de Tarascon-sur-Ariège, Préhistoire ariègeoise, Bull. de la Société préhistorique Ariège-Pyrénées, t. 40, p. 71-87, 8 fig.

SIMONNET R., 1996 - Approvisionnement en silex au Paléolithique supérieur : déplacements et caractéristiques physionomiques des paysages, l'exemple des Pyrénées centrales, in : DELPORTE H. et CLOTTES J. (dir.), Pyrénées Préhistoriques - Arts et Sociétés, actes du 118e congrès national des sociétés historiques et scientifiques, Pau 1993, Editions du CTHS, p. 117-128, 10 fig.

SIMONNET R., 1999a - Le silex et la fin du Paléolithique supérieur dans le bassin de Tarascon-surAriège, Préhistoire ariégeoise, Bull. de la Société préhistorique Ariège-Pyrénées, 1998, t. LIII, p. 181-222, 6 fig., 4 tabl.

SIMONNET R., 1999b - De la géologie à la Préhistoire : le silex des Prépyrénées. Résultats et réflexions sur les perspectives et les limites de l'étude des matières premières lithiques, Paléo, 11, p. 71-88, 14 fig., 4 tableaux.

\section{NOTES}

1. Le site de Las Sablas se situe sur la terrasse FCx de la vallée de l'Aube. Cette terrasse est formée d'une couche de limon surmontant des graviers. L'Aube y a surcreusé son cours actuel, s'enfonçant dans ses propres graviers. Ceux-ci sont ainsi visibles tout le long du lit. Il a été possible d'observer que, descendus de Visker, ils ne renferment pas de galets de silex.Les blocs de silex n'existent qu'au sommet du limon et en surface, ce qui laisse supposer un apport latéral par glissement depuis le bois du Bécut et le haut de la Décharge. D'autre part, il est à noter que 
l'industrie qui accompagne ces blocs est limitée au seul Paléolithique supérieur. Si Paléolithique moyen il y a, il ne peut être que sur les graviers ou à la base du limon.

2. L'occupation moustérienne est attestée sur tous les secteurs d'Hibarette. Quelques bifaces de bonne facture, de nombreux racloirs de tous types, des denticulés et des coches sont présents. Les nucléus issus du débitage Discoïde (généralement dans sa modalité bifaciale) et une composante de nucléus Levallois (récurrents centripètes dominants) constituent un part importante des récoltes des prospections. L'utilisation du silex de Montgaillard/Hibarette est aussi attestée au Paléolithique moyen dans de nombreuses stations périphériques.

3. Ce site a été découvert simultanément par J. Barragué, J.-M. Cardeilhac et $\mathrm{C}$. Rousseau.

4. Ces trois sites sont inventoriés selon une numérotation spécifique qui renvoie à la lithothèque régionale. Ils portent respectivement les numéros : 09181 01, 09094 04, 3108601.

\section{RÉSUMÉS}

Les auteurs présentent les résultats d'une étude portant sur le silex du flysch de Montgaillard et sur les ateliers de plein air paléolithiques de Hibarette (Hautes-Pyrénées) où il a été exploité. L'acquisition des données s'est faite par le biais d'une prospection systématique étalée sur une vingtaine d'années. La matière première a fait l'objet d'une caractérisation pétrographique et le contexte géomorphologique des gîtes exploités a pu être précisé.

Les ateliers de taille ont fonctionné à toutes les périodes de la Préhistoire : Paléolithique moyen, supérieur et Néolithique. L'exploitation du silex au Paléolithique supérieur semble se placer surtout à l'Aurignacien, au Solutréen et au Magdalénien (ancien, moyen/supérieur). Néanmoins, il existe quelques indices de passages au Châtelperronien, au Gravettien ainsi qu'à l'Epipaléolithique. De par la qualité du silex, la masse de matière première qui a été débitée sur les ateliers de taille, et sa diffusion dans toutes les Pyrénées, le silex de Montgaillard/Hibarette tient une place de choix dans l'économie des matières siliceuses des Pyrénées centrales et les gîtes/ateliers de Hibarette sont certainement parmi les plus importants de cette région.

The authors present the results of a study about both the flint of the Montagaillard flysch, but also about the Palaeolithic open air workshops in Hibarette (Hautes-Pyrénées) where the results have been analyzed. Data have been provided thanks to systematic surveys over twenty years. Raw material has been analyzed through a petrographical characterization and the geomorphological profile of the studied locations has been described as well. Knapping workshops have been used all though Prehistory time, that is to say Middle and Upper Palaeolithic, but also Neolithic.

Flint production at Upper Palaeolithic time seems to correlate with Aurignacian, Solutrean and Magdalenian (old, Middle and Upper). However, there are some pieces of evidence for an adequation with Chatelperronian, Gravettian and Epipalaeolithic.

Thanks to the flint quality, the quantity of raw material removed in knapping workshops, and its presence overall in the Pyrénées, Montgaillard/Hirabette flint is very well positionned among siliceous materials trade of Central Pyrénées; flint workshops of Hirabette are certainly part of the most important ones in this area. 
INDEX

Mots-clés : ateliers de taille, gîtes de matières premières, Paléolithique supérieur, Pyrénées, silex du flysch

Keywords : flysch flint, knaping workshops, Pyrénées, raw material deposits, Upper Palaeolithic

\section{AUTEURS}

MARC JARRY

UMR 5608, UTAH, Maison de la Recherche, Université de Toulouse-le-Mirail PASCAL FOUCHER

UMR 5608, UTAH, Maison de la Recherche, Université de Toulouse-le-Mirail ROBERT SIMONNET

UMR 5608, UTAH, Maison de la Recherche, Université de Toulouse-le-Mirail 